\title{
What is the functional role of delay-related alpha oscillations during working memory?
}

Michael J. Wolff ${ }^{1 *}$, Elkan G. Akyürek², Mark G. Stokes ${ }^{1 *}$

${ }^{1}$ Department of Experimental Psychology, University of Oxford, Oxford, UK

${ }^{2}$ Department of Experimental Psychology, University of Groningen, Groningen, The Netherlands

*Corresponding authors: $\quad \underline{\text { michael.wolff@psy.ox.ac.uk }}$ mark.stokes@psy.ox.ac.uk
"Activity-silent" working memory (WM) has been proposed as an energy-efficient short- term storage mechanism that does not require persistent spiking ${ }^{1}$. Previously we developed a novel experimental approach to test its key predictions and showed that an external perturbation results in a subtle but reliable readout of the information in WM ${ }^{2,3}$, even for unattended memories that otherwise do not exhibit persistent neural activity during maintenance ${ }^{4}$. More recently, there has been intense interest in what differentiates attended and unattended items in WM, with diverse suggestions ranging from storage in different brain areas ${ }^{5}$, reversed neural codes ${ }^{6}$ or that both are stored in low-frequency oscillatory activity. Here we specifically consider this latter suggestions, as proposed by Barbosa et al. ${ }^{7}$ based on their secondary analysis of our freely available EEG data ${ }^{2,3}$. We are pleased that our previously published data have contributed to new and potentially important findings, but we also note that this oscillatory correlate raises further questions, and does not necessarily disqualify activity-silent models of WM.

Alpha oscillations are ubiquitous in human EEG, and correlate with a multitude of cognitive processes ${ }^{8}$. It is unclear whether WM content decoding in alpha power signifies a functional role for WM maintenance, or reflects a correlated cognitive process like spatial attention ${ }^{9}$, or even just spontaneous activity in a temporary patterned network ${ }^{10}$. Furthermore, alpha decoding results for unattended WM content can produce inconsistent results. While Barbosa et al. ${ }^{7}$ report alpha decoding of the unattended item in our data, Rose et al. ${ }^{11}$ found no sustained alpha code for unattended WM content (see also ${ }^{12}$ ). Further clarity on the circumstances required for decodable alpha will be important for establishing a functional role in WM.

A separate issue raised by Barbosa et al. ${ }^{7}$ is that the impulse effect could be due to a stimulus evoked reducing in neural noise that transiently unmasks previously weak signals. 
We follow-up on this proposal by exploring whether the decodable alpha power prior to impulse-onset (whatever its functional significance) may help generate the impulse response effect, for example via phase reset ${ }^{13}$. This could explain why alpha power decoding seems to work better than raw EEG voltage traces during the delay (where alpha oscillations are not time-locked), and explain the decrease in across-trial variance after impulse presentation, when oscillations are time-locked (see Fig. 2). However, in contrast to this prediction, decoding WM from impulse-evoked alpha power was relatively inconsistent across experiments in ${ }^{2,3}$ (Fig. 1a,b). Moreover, even when evoked power decoding was present, the discriminative spatial patterns were not statistically related to the discriminate patterns associated with induced alpha power before impulse onset (Fig. 1c), which should have been the case if the decodable impulse response was purely due to a phase reset. Thus, while neural oscillations have been shown to modulate evoked responses in general ${ }^{14}$ and even the impulse response specifically ${ }^{15}$, we have found no evidence that decodable alpha oscillations cause the WM-specific impulse response.
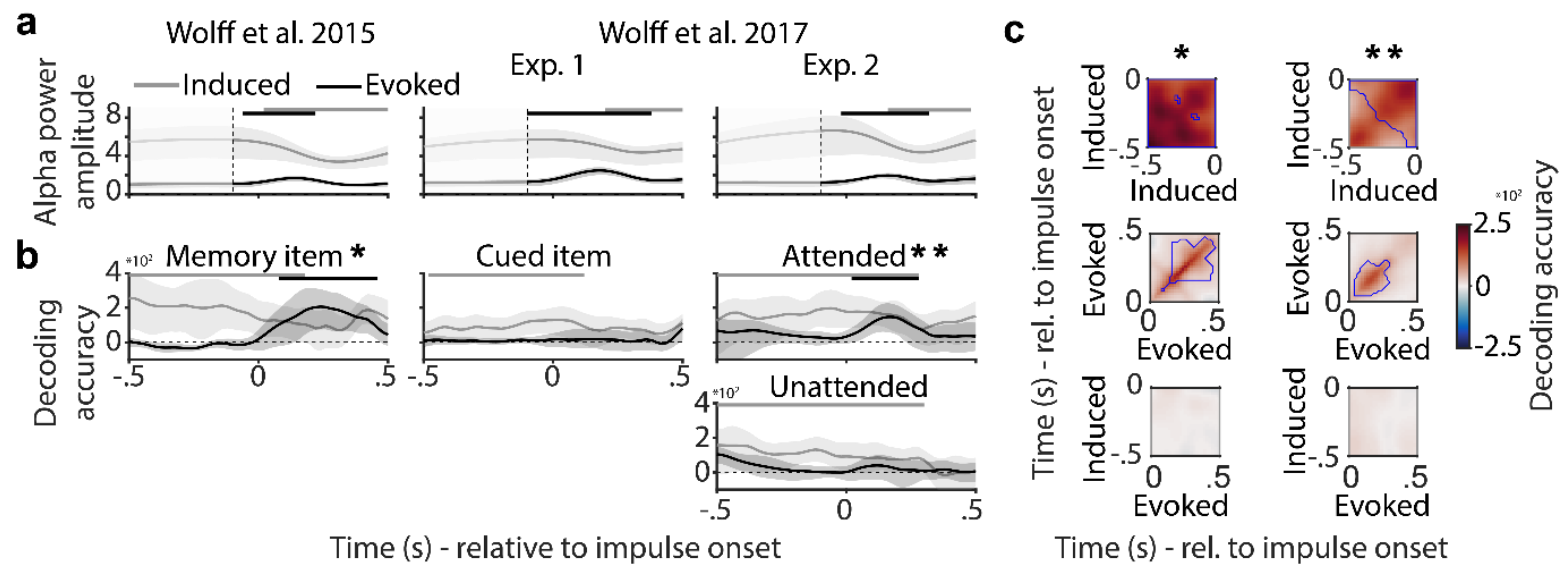

Figure 1. No evidence that induced alpha power mediates impulse-evoked decoding in 2,3 . (a) Induced (grey) and evoked (black) alpha power amplitude. Significant modulations relative to pre-impulse amplitude (-.5 to $-.1 \mathrm{~s}$, dashed vertical line) are represented by the upper bars in the corresponding colours ( $p<0.05$, cluster-corrected, two-sided). The presentation of the impulse stimulus triggers an increase in evoked and a decrease in induced alpha power. (b) Decoding accuracies of memory items for both induced (grey) and evoked (black) power. Above-chance decoding is indicated by the upper bars $(p<0.05$, cluster-corrected, one-sided). While induced alpha power decoding roughly mirrors induced alpha power amplitude (decrease after impulse in all cases), evoked power decoding is only present after impulse presentation in two instances (for the memory item in ${ }^{3}$ and for the attended item of experiment 2 in ${ }^{2}, *$ and ${ }^{* *}$, respectively). Error-shading, $95 \% \mathrm{Cl}$. of the mean. (c) Cross-temporal follow-up analyses when both induced and evoked alpha decoding were present (* memory item in ${ }^{3}$, left column; ** attended item of exp. 2 in ${ }^{2}$, right column ). Significant cross-temporal decoding clusters are outlined in blue $(p<0.05$, clustercorrected, two-sided). Induced power pre-impulse decoding (top) and evoked power postimpulse decoding (middle) show no evidence of cross-generalization (bottom). 
Barbosa et al. ${ }^{7}$ further argue that the impulse-induced variance reduction is incompatible with their own activity-silent WM model ${ }^{16}$, where an increase in variance is predicted when 'hidden' WM content is reactivated "in some trials". Firstly, the activity-silent model we propose does not require impulse-triggered 'reactivation' or 'transformation' of unattended to attended in some trials; rather the impulse provides a subtle but reliable readout of the WM state in each trial ${ }^{2}$. The impulse can thus also be used to investigate the WM states of actively maintained WM items, which, as pointed out by Barbosa et al. ${ }^{7}$, has provided evidence for diffusing dynamics ${ }^{17}$. Notably, this finding is in line with silent WM models in which items are periodically refreshed with transient activity bursts ${ }^{18,19}$, leading to a similar error accumulation (i.e., drift) as predicted by purely activity based models ${ }^{20}$. Secondly, the observation of an increase in variance in the EEG after transcranial magnetic stimulation (TMS) in ${ }^{11}$, while intriguing, should be interpreted with caution, as TMS-induced artefacts are difficult to remove completely ${ }^{21,22}$, and likely leave behind a noisy voltage trace. Finally, it is well known that visual stimulation temporarily reduces alpha power ${ }^{23}$. This alpha reduction may not only be linked to the drop in induced alpha-power decoding in our data (Fig. 1b), but also seems to be a main contributor to the post-impulse variance reduction (Fig. 2a \& b). Indeed, low pre-impulse alpha-power trials exhibit almost no impulse-induced decrease in variance (in fact an increase is observable in some cases). Critically, if the impulse effect was simply due to noise reduction mediated by alpha power reduction, then trials with low alpha power (and therefore low across-trial variance) should exhibit higher WM content decoding from the voltage trace during the delay period. However, we found no such relationship between alpha-power and decoding accuracy (Fig. 2c). 


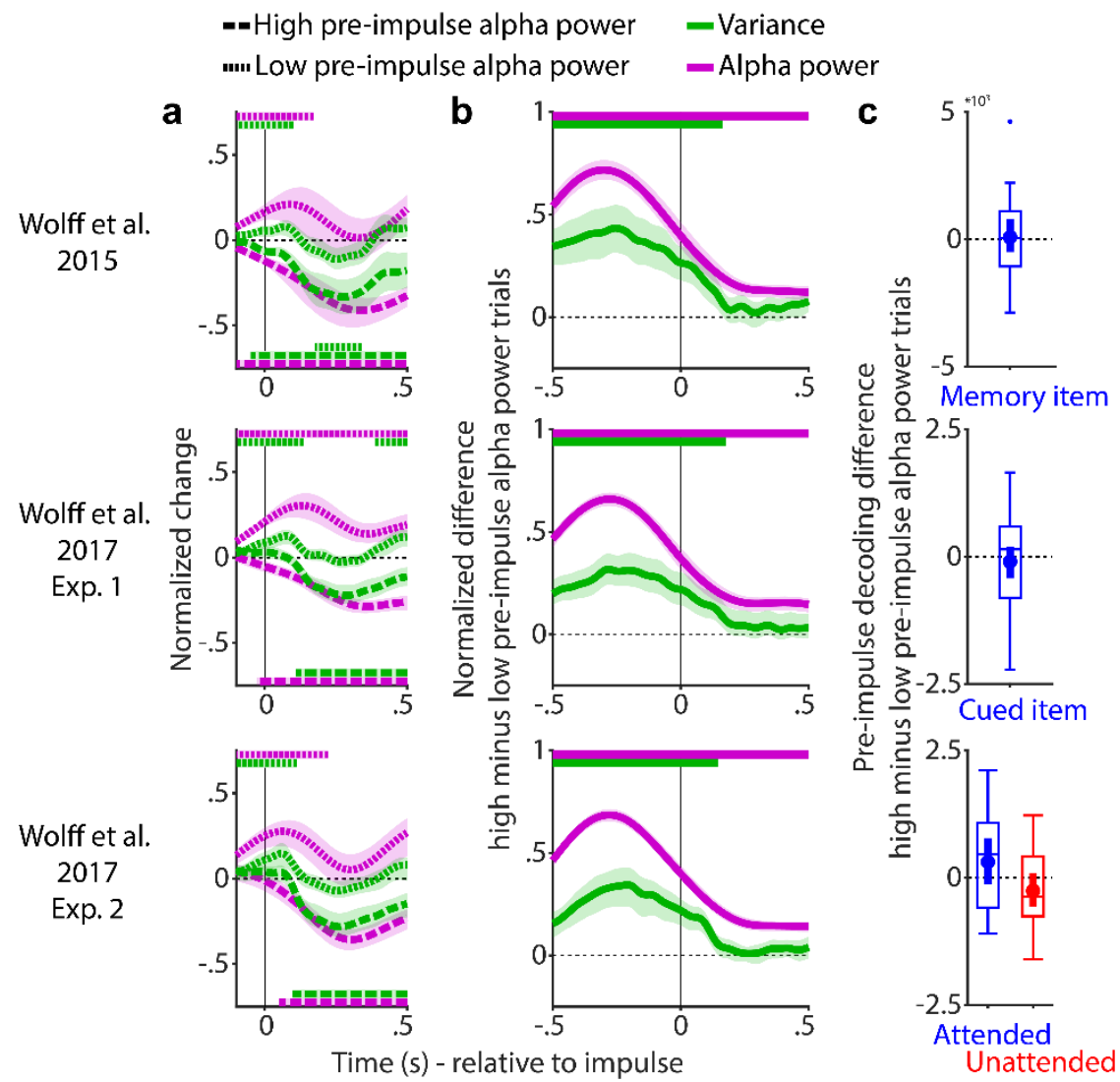

91 Figure 2. Impulse-induced alpha power reduction is associated with a reduction in trial-wise 92 variance but does not correlate with voltage-based decoding. (a) Normalized change from pre-impulse period (-.5 to -.1 s for alpha power; -.5 to $0 \mathrm{~s}$ for variance) in alpha power (pink) and across-trial voltage variance (green), separately for high (long dashes) and low (short dashes) pre-impulse (-.5 to -.1 s) alpha power trials. Significant increases and decreases are represented by bars in the corresponding appearances at the top and bottom, respectively ( $p<0.05$, cluster-corrected, two-sided). Alpha and variance changes after impulse onset show are similar and are modulated by pre-impulse alpha power; variance reduction is almost non-existent in high alpha power trials. Error-shading, $95 \% \mathrm{Cl}$. of the mean. (b) Normalized difference between high and low pre-impulse (-.5 to -.1 s) alpha power trials in variance (green) and alpha power (purple). Significant differences are indicated by bars in the corresponding colours ( $p<0.05$, cluster-corrected, two-sided). Higher alpha power trials also exhibit higher variance than low alpha power trials. The variance difference disappears after impulse onset. (c) No evidence for a difference in pre-impulse decoding accuracy from the voltage trace $(-5$. to $0 \mathrm{~s}$ ) between high and low pre-impulse alpha-power trials (-.5 to -.1 s). Circles and error bars represent the mean and $95 \% \mathrm{Cl}$. of the mean, respectively. 
107 Barbosa et al. ${ }^{7}$ point out that according to the synaptic WM model, any stimulus, no matter

108 its behavioural relevance, leaves behind a synaptic trace ${ }^{1}$, and have shown in their own

109 research that these traces may cause the behavioural bias toward no longer relevant 110 information ${ }^{16}$ (i.e. serial dependency ${ }^{24}$ ). They reason this is at odds with our original 111 observation that the impulse response is unrelated to an uncued WM item ${ }^{2}$. However, we 112 argue that the time-course of our finding, where the impulse was presented $1.1 \mathrm{~s}$ after the 113 item was rendered irrelevant, is consistent with models of short-term synaptic plasticity that 114 posit short-term connectivity changes dissipate within 1 to 2 seconds when not reinforced

1151,25 . Alternatively, it is also possible that no-longer relevant information could be actively 116 removed from the hidden WM state to free up limited resources. We also turn to the serial 117 dependency effect to show that the behavioural bias toward previous trials is specific to 118 cued items. We find no evidence of a bias toward a previously uncued item, even when it is closer in time than the previously cued item (Supplementary fig. 1; see also ${ }^{26,27}$ ). These results highlight the flexibility of WM and a potential distinction between actively and passively removed WM content. We propose that while passive removal may leave behind a neural trace in the WM network, active removal may not.

123 Whether or not WM maintenance relies on persistent neural firing is an important and 124 ongoing issue. On one side of the debate, researchers point to evidence of activity 125 correlates ${ }^{28}$, and on the other side, researchers have noted activity gaps ${ }^{18}$. Correlates may be epiphenomena and gaps may be null effects. Barbosa et al. ${ }^{7}$ rightly point out that absence of evidence of activity-based coding for unattended items does not prove the existence of activity-silent storage. However, despite their claim to the contrary, the same is logically true for presence of an activity signature: it may not be a functional or sufficient mechanism for WM maintenance. For instance, even if ongoing activity is not completely epiphenomenal, it may only reflect periodic refreshing of the network during maintenance. This may also explain why persistent activity such as that currently observed in the alpha band is quite weak overall: the connectivity in the underlying neural network may be its scaffold. Thus, WM could still be mediated by activity-silent mechanisms, even if there is a decodable correlate in alpha.

To conclude, while we agree that more work is needed to establish synaptic plasticity as a necessary and sufficient mechanism for maintenance in WM, we also argue that further work is similarly needed to establish the same for this alternative mechanism proposed by Barbosa et al. ${ }^{7}$ However, we are confident that complementary research at different levels of analysis, and using both measuring and perturbations, will continue yielding important insights to the underlying neural mechanisms of WM. 


\section{Author contributions}

143 MJW analysed the data. MJW and MGS wrote the first manuscript draft. MJW, EGA, and

144 MGS edited and reviewed the manuscript.

145

146 Competing interests

147 The authors declare no competing interests.

148

149 Data availability

150 All code and data (that is not already openly available) will be available after peer-reviewed 151 publication at osf.io/e7k2a.

152

\section{Acknowledgments}

154 This research was in part funded by a James S. McDonnell Foundation Scholar Award 155 (220020405) to MGS and an Open Research Area grant to MGS (ESRC ES/S015477/1) and 156 EGA (NWO 464.18.114). The Wellcome Centre for Integrative Neuroimaging is supported by 157 core funding from the Wellcome Trust (203139/Z/16/Z). The views expressed are those of 158 the authors and not necessarily those of the National Health Service, the National Institute 159 for Health Research or the Department of Health. The funders had no role in study design, 160 data collection and analysis, decision to publish, or preparation of the manuscript. 
1. Mongillo, G., Barak, O. \& Tsodyks, M. Synaptic Theory of Working Memory. Science 319, 1543-1546 (2008).

2. Wolff, M. J., Jochim, J., Akyürek, E. G. \& Stokes, M. G. Dynamic hidden states underlying working-memory-guided behavior. Nature Neuroscience 20, 864-871 (2017).

3. Wolff, M. J., Ding, J., Myers, N. E. \& Stokes, M. G. Revealing hidden states in visual working memory using electroencephalography. Front. Syst. Neurosci. 9, (2015).

4. Lewis-Peacock, J. A. \& Postle, B. R. Decoding the internal focus of attention. Neuropsychologia 50, 470-478 (2012).

5. Christophel, T. B., lamshchinina, P., Yan, C., Allefeld, C. \& Haynes, J.-D. Cortical specialization for attended versus unattended working memory. Nature Neuroscience 21, 494 (2018).

6. van Loon, A. M., Olmos-Solis, K., Fahrenfort, J. J. \& Olivers, C. N. Current and future goals are represented in opposite patterns in object-selective cortex. eLife 7, (2018).

7. Barbosa, J., Soldevilla, D. L. \& Compte, A. Unattended short-term memories are maintained in active neural representations. (2021) doi:10.31234/osf.io/qv6fu.

8. Clayton, M. S., Yeung, N. \& Kadosh, R. C. The many characters of visual alpha oscillations. European Journal of Neuroscience 48, 2498-2508 (2018).

9. Bae, G.-Y. \& Luck, S. J. Dissociable Decoding of Spatial Attention and Working Memory from EEG Oscillations and Sustained Potentials. J. Neurosci. 38, 409-422 (2018).

10. Sugase-Miyamoto, Y., Liu, Z., Wiener, M. C., Optican, L. M. \& Richmond, B. J. Short-Term Memory Trace in Rapidly Adapting Synapses of Inferior Temporal Cortex. PLOS Comput Biol 4, e1000073 (2008).

11. Rose, N. S. et al. Reactivation of latent working memories with transcranial magnetic stimulation. Science 354, 1136-1139 (2016).

12. LaRocque, J. J., Lewis-Peacock, J. A., Drysdale, A. T., Oberauer, K. \& Postle, B. R. Decoding Attended Information in Short-term Memory: An EEG Study. Journal of Cognitive Neuroscience 25, 127-142 (2012).

13. HansImayr, S. et al. Alpha Phase Reset Contributes to the Generation of ERPs. Cereb Cortex 17, 1-8 (2007).

14. lemi, L. et al. Multiple mechanisms link prestimulus neural oscillations to sensory responses. elife 8, e43620 (2019).

15. ten Oever, S., De Weerd, P. \& Sack, A. T. Phase-dependent amplification of working memory content and performance. Nature Communications 11, 1832 (2020).

16. Barbosa, J. et al. Interplay between persistent activity and activity-silent dynamics in the prefrontal cortex underlies serial biases in working memory. Nature Neuroscience 23, 1016-1024 (2020).

17. Wolff, M. J., Jochim, J., Akyürek, E. G., Buschman, T. J. \& Stokes, M. G. Drifting codes within a stable coding scheme for working memory. PLOS Biology 18, e3000625 (2020). 
18. Lundqvist, M., Herman, P. \& Miller, E. K. Working Memory: Delay Activity, Yes! Persistent Activity? Maybe Not. J. Neurosci. 38, 7013-7019 (2018).

19. Lundqvist, M. et al. Gamma and Beta Bursts Underlie Working Memory. Neuron 90, 152-164 (2016).

20. Wimmer, K., Nykamp, D. Q., Constantinidis, C. \& Compte, A. Bump attractor dynamics in prefrontal cortex explains behavioral precision in spatial working memory. Nat Neurosci 17, 431-439 (2014).

21. Mutanen, T. P. et al. Recovering TMS-evoked EEG responses masked by muscle artifacts. Neurolmage 139, 157-166 (2016).

22. Casula, E. P. et al. TMS-evoked long-lasting artefacts: A new adaptive algorithm for EEG signal correction. Clinical Neurophysiology 128, 1563-1574 (2017).

23. Min, B.-K. et al. The best of both worlds: Phase-reset of human EEG alpha activity and additive power contribute to ERP generation. International Journal of Psychophysiology 65, 58-68 (2007).

24. Fischer, J. \& Whitney, D. Serial dependence in visual perception. Nat Neurosci 17, 738743 (2014).

25. Zucker, R. S. \& Regehr, W. G. Short-Term Synaptic Plasticity. Annu. Rev. Physiol. 64, 355405 (2002).

26. Fischer, C. et al. Context information supports serial dependence of multiple visual objects across memory episodes. Nature Communications 11, 1932 (2020).

27. Czoschke, S., Fischer, C., Beitner, J., Kaiser, J. \& Bledowski, C. Two types of serial dependence in visual working memory. British Journal of Psychology 110, 256-267 (2019).

28. Constantinidis, C. et al. Persistent Spiking Activity Underlies Working Memory. J. Neurosci. 38, 7020-7028 (2018). 
225 Supplementary Information

226 Supplementary Fig. 1

227 Supplementary Methods

228 Supplementary References 

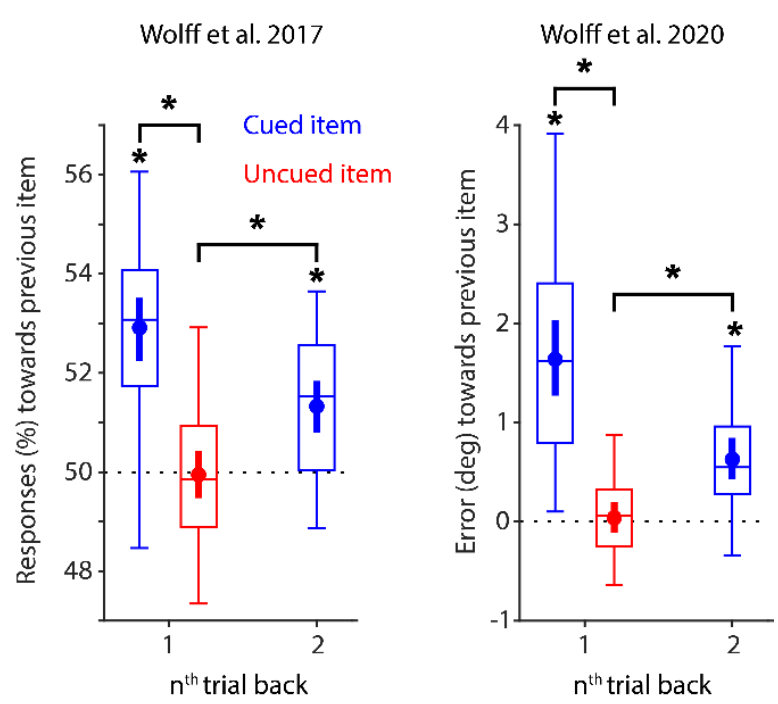

230 Supplementary figure 1. Serial dependency in two retro-cue experiments in ${ }^{1}$ (forced choice; left) and ${ }^{2}$ (free recall; right). Clear serial dependence towards the previously cued item (for both 1 and 2 trials back) was found in both experiments but none towards the previously uncued item. This suggests that retro-cues may enable an active removal of WMcontent, which eliminates serial dependency by potentially wiping the synaptic trace. Significant above-chance responses (left) and report-errors (right) towards either the previously cued (blue) or uncued (red) items are indicated with asterisks. Significant pairwise differences in serial dependence between the 1-back uncued and 1-back and 2back cued item are also indicated with asterisks $(p<0.05$, cluster-permutation-test, twosided; planned comparisons, not corrected). Circles and error bars represent the mean and $95 \% \mathrm{Cl}$. of the mean, respectively. 


\section{Experimental datasets}

243

244

245

246

247

248

249

250

251

252

253

We focused our main reanalyses on the EEG datasets of the three experiments in ${ }^{1,3}$. We did not use the openly available, preprocessed and epoched datasets, but rather adjusted the preprocessing slightly using our original raw EEG data (see EEG preprocessing below). In 3 participants $(n=24)$ completed a one-item WM task. In experiment 1 of ${ }^{1}$ participants $(n=30)$ completed a two-item retro-cue WM task, where the retro-cue indicated with $100 \%$ accuracy which item was probed at the end of each trial. In experiment 2 of ${ }^{1}$ participants $(n=19)$ completed a two-item WM task, where both items were serially probed in a predictable order, thus resulting in attentional shifts between items. The memory items in all three experiments of ${ }^{1,3}$ were orientation gratings and memory recall were forced-choice. Additionally, we reanalysed the behavioural data of ${ }^{2}(n=26)$, which was a free-recall retrocue task.

\section{EEG preprocessing}

The preprocessing pipeline for the datasets in ${ }^{1,3}$ remained largely the same as in the original articles. The only differences are that we ensured that the sample rate was the same in all three datasets $(500 \mathrm{hz}$ ), voltage traces were not baselined (unless specifically mentioned), and trials were epoched relative to the impulses from - .9 to $.9 \mathrm{~s}$ in all cases (note that we only considered the first impulse in experiment 2 of $\left.{ }^{1}\right)$. As in the original articles, we only included the 17 posterior electrodes (P7, P5, P3, P1, Pz, P2, P4, P6, P8, PO7, PO3, POz, PO4, $\mathrm{PO}$, $\mathrm{O} 1, \mathrm{Oz}, \mathrm{O} 2)$ in all subsequent EEG analyses.

\section{Alpha power decomposition}

Alpha power amplitude was computed relative to impulse onsets from - .5 to $.5 \mathrm{~s}$. The EEG voltage data was filtered between 8 to $12 \mathrm{~Hz}$ and Hilbert transformed using the following code in Matlab:

$$
\text { abs(hilbert(eegfilt(data, hz, 8, 12))) }
$$

where data is the voltage time-course of a single channel, $h z$ is the sample-rate of the data $(500 \mathrm{hz})$, and 8 and 12 are the low and high frequency cut-offs, respectively.

Induced alpha power was obtained by computing the alpha power of each trial individually, whereas evoked alpha power was obtained by first averaging the voltage traces of a subset of trials within each orientation bin (see decoding analysis below) and then computing the alpha power on those averaged subset of trials. The number of trials that were used for averaging was slightly different for each experiment and subject and depended on the total number of trials per subject (minimum number of trials for averaging per experiment: $M=$ 24.83, $S D=2.32$ in $^{3}, M=20.3, S D=2.1$ in experiment 1 of ${ }^{1}$, and $M=31.42, S D=3$ in experiment 2 of ${ }^{1}$ ). To explore the time-course of induced/evoked alpha power from -.5 to .5 $s$ relative to impulse, the induced/evoked power was averaged across electrode and trials/subset of trials for each participant. 
We used induced and evoked alpha power to decode WM content in each experiment of 1,3 from - .5 to $.5 \mathrm{~s}$ relative to impulse onset. First, trials were assigned the closest of eight orientation bins. The data was then randomly split into three folds using random stratified sampling, ensuring a balanced distribution of orientations across folds. Same orientation-bin trials were then averaged within each fold, resulting in 24 pseudo-trials, one pseudo-trial per orientation-bin in each fold. Note that this averaging was done after obtaining trial-wise alpha-power in the case of induced alpha-power decoding, but before (and hence on the voltage traces) in the case of evoked alpha-power decoding. Using cross-validation, the classifier was trained on the pseudo-trials of two train-folds at a specific time-point, and tested on the left-out test-fold at the same point. The covariance matrix was computed from the pseudo-trials in train-folds using a shrinkage estimator ${ }^{4}$. The pseudo-trials of the train-folds were then averaged within each orientation bin, resulting in one exemplar per bin. The average bins were convolved with a half cosine basis set raised to the $7^{\text {th }}$ power to pool information across neighbouring orientations ${ }^{5}$. The Mahalanobis distances between each pseudo-trial of the left-out test-fold and the eight averaged pseudo-trials of the trainfolds were sign-reversed and ordered to obtain a similarity profile. This was summarized into a single decoding value by computing the cosine vector mean, where a higher value suggests a higher pattern similarity between similar orientations ${ }^{1,6}$. The above procedure was repeated 20 times, with randomly split folds each time, separately for eight orientation spaces $\left(0^{\circ}\right.$ to $168.75^{\circ}, 1.40625^{\circ}$ to $170.1563^{\circ}, 2.8125^{\circ}$ to $171.5625^{\circ}, 4.2188^{\circ}$ to $172.9688^{\circ}$, $5.625^{\circ}$ to $174.375^{\circ}, 7.0313^{\circ}$ to $175.7813^{\circ}, 8.4375^{\circ}$ to $177.1875^{\circ}, 9.8438^{\circ}$ to $178.5938^{\circ}$, each in steps of $22.5^{\circ}$ ), and then averaged across all iterations. This procedure was done separately for each time-point from - .5 to $.5 \mathrm{~s}$ in steps of $0.02 \mathrm{~s}$ relative to impulse onsets.

We were also interested if and to what extent potential pre-impulse induced alpha power decoding matches potential post-impulse evoked alpha power decoding. A crossgeneralization between those two patterns would suggest that the memory-specific impulse effect is the result of previous memory-specific oscillations. We thus explored the crosstemporal generalization profiles of pre-impulse induced power decoding, post-impulse evoked power decoding, as well as the generalization between them. A prerequisite for this was that the analysis described in the paragraph above found significant pre-impulse induced and significant post-impulse evoked power decoding. The decoding approach was similar as the one described above, but the classifier was trained and tested on all possible time-point combinations within a window of -.5 to $0 \mathrm{~s}$, for induced alpha power, and 0 to .5 $\mathrm{s}$ for evoked alpha power. To test for generalization between them, the classifier was trained on pre-impulse induced power $(-.5$ to $0 \mathrm{~s})$ and tested on post-impulse evoked power (0 to $.5 \mathrm{~s})$, for all possible time-point combinations.

317 We also used the voltage trace during the delay for WM content decoding (from -.5 to $0 \mathrm{~s}$, in steps of $0.002 \mathrm{~s}$ ). Here, the voltage trace was first baselined by removing the average voltage from -.2 to $0 \mathrm{~s}$ relative to memory array onset. We used the same approach as in our original article ${ }^{1}$, which resulted in trial-wise decoding values. 
324 We obtained the across-trial variances for each subject relative to -.5 to $.5 \mathrm{~s}$ at each timepoint relative to impulse onsets using the trial-wise voltage traces averaged across all 17 electrodes. The across-trial variance time-course was smoothed with a gaussian kernel ( $S D=$ $20 \mathrm{~ms}$ ). Unlike in ${ }^{7}$, we did not detrend or baseline the voltage traces beforehand to not distort the time-course of potential variance fluctuations before and after the impulse, but removed extreme trials with absolute average voltages that exceeded 100 microvolts. Note that this lead to the removal of trials for only a few participants (two trials of one participant in ${ }^{3} ; 17$ trials of one participant in experiment 1 of ${ }^{1}$; three and one trial(s) for two participants in experiment 2 of ${ }^{1}$ ). To test the relationship between pre-impulse induced alpha power and across-trial variance, trials were median-split into two sets based on the trial-wise induced alpha power averaged from - .5 to - $.1 \mathrm{~s}$ relative to impulse. The across-trial variance was obtained separately for each set. Normalized induced alpha power change and across-trial variance change after impulse was obtained separately for each set using the same formula as in ${ }^{7}$ :

where data and base_data was either across-trial variance and pre-impulse across-trial variance $(-.5$ to $0 \mathrm{~s})$, respectively, or induced alpha power and pre-impulse induced alpha power $(-.5$ to $-.1 \mathrm{~s})$, respectively.

\section{Relationship between induced alpha power and voltage decoding}

To test if delay alpha power is related to delay voltage decoding, the trial-wise decoding values (averaged from -.5 to $0 \mathrm{~s}$ relative to impulse) were median-split based on the trialwise induced alpha power (-.5 to -.1 s relative to impulse). For experiment 1 and 2 in ${ }^{1}$ this was done separately for location condition and session, respectively. The differences between the averaged splits were tested for statistical significance against 0 .

Serial dependency

We tested if memory reports in the forced-choice retro-cue experiment in ${ }^{1}$ and the freerecall retro-cue experiment in ${ }^{2}$ show evidence of a systematic bias towards both the cued and the uncued memory item of the previous trial (i.e. serial dependency ${ }^{8}$ ). We removed trials with previous inter-trial-intervals (from previous response to current memory array onset) longer than $10 \mathrm{~s}$ for 1-back, and trials with previous inter-trial-intervals that were cumulatively longer than $20 \mathrm{~s}$ for 2-back serial dependency investigations. We further removed trials with report errors larger than 3 circular standard deviations in the free-recall experiment (in total removed trials in forced-choice, 1-back: $M=2.42 \%, S D=1.76 \%$; 2 back: $M=3.09 \%, S D=2.64 \%$; free-recall, 1-back: $M=7.34 \%, S D=2.54 \%$; 2 -back: $M=$ $9.48 \%, S D=3.12 \%)$.

359 In the forced choice experiment, subjects were instructed to report if the cued item was rotated clockwise $(\mathrm{CW})$ or counter-clockwise (CCW) relative to the probe. We quantified serial-dependency by computing the percentage of responses that matched the direction ( $\mathrm{CW}$ or $\mathrm{CCW}$ ) of the relative difference between the previous item in question (cued or uncued) and the current memory item. The reasoning for this was that if the previous item 
was $\mathrm{CCW}$ relative to the current, it may pull the memory of the latter further $\mathrm{CCW}$ and therefore increase the likelihood of reporting CCW to the probe.

366 For the free-recall experiment, we similarly obtained the magnitude of report error that was in the same direction as the difference between the current and the previous item in question. We simply sign-reversed the relative error of all trials where the previous item in question was rotated CCW relative to the current item, before taking the average of relative errors across all trials for each subject.

371 In both cases, we tested the influence of the previously cued item on the report of the

372 current at both 1 trial and 2 trials back and the influence of the uncued item at 1 trial back.

\section{$373 \quad$ Statistical significance testing}

374 We used the sign-permutation test, which does not make assumptions about the underlying 375 data distribution, to determine the statistical significance in all cases. Null distributions for 376 each test were obtained by randomly flipping the sign of each subject's data (e.g. decoding

377 value, variance, alpha power, serial dependence, or difference between conditions) 100,000 378 times. The distribution of each test was then used to obtain the $P$ value of the null

379 hypothesis that there was no effect (i.e. mean effect of 0 ) or no difference between 380 compared conditions. For time-series data, the above procedure was applied to each timepoint separately, and subsequently corrected for multiple comparisons with a cluster-based permutation test with 100,000 permutations and a cluster-forming threshold of $P<0.05$. The significance threshold of all tests was set at $P<0.05$. Tests on one-dimensional decoding results obtained after training and testing on the same time-points using crossvalidation were one-sided $(>0)$, since negative decoding results are uninterpretable in those cases. All other tests (including tests on cross-temporal decoding matrices) were two-sided. 
1. Wolff, M. J., Jochim, J., Akyürek, E. G. \& Stokes, M. G. Dynamic hidden states underlying working-memory-guided behavior. Nature Neuroscience 20, 864-871 (2017).

2. Wolff, M. J., Jochim, J., Akyürek, E. G., Buschman, T. J. \& Stokes, M. G. Drifting codes within a stable coding scheme for working memory. PLOS Biology 18, e3000625 (2020).

3. Wolff, M. J., Ding, J., Myers, N. E. \& Stokes, M. G. Revealing hidden states in visual working memory using electroencephalography. Front. Syst. Neurosci. 9, (2015).

4. Ledoit, O. \& Wolf, M. Honey, I shrunk the sample covariance matrix. The Journal of Portfolio Management 30, 110-119 (2004).

5. Brouwer, G. J. \& Heeger, D. J. Decoding and reconstructing color from responses in human visual cortex. J Neurosci 29, 13992-14003 (2009).

6. Sprague, T. C., Ester, E. F. \& Serences, J. T. Restoring Latent Visual Working Memory Representations in Human Cortex. Neuron 91, 694-707 (2016).

7. Barbosa, J., Soldevilla, D. L. \& Compte, A. Unattended short-term memories are maintained in active neural representations. (2021) doi:10.31234/osf.io/qv6fu.

8. Fischer, J. \& Whitney, D. Serial dependence in visual perception. Nat Neurosci 17, 738743 (2014). 